\title{
Thermal Spin-Transfer Torque in Magnetoelectronic Devices
}

\author{
Moosa Hatami and Gerrit E. W. Bauer \\ Kavli Institute of NanoScience, Delft University of Technology, Lorentzweg 1, 2628 CJ Delft, The Netherlands \\ Qinfang Zhang and Paul J. Kelly \\ Faculty of Science and Technology and MESA+ Institute for Nanotechnology, University of Twente, P.O. Box 217, \\ 7500 AE Enschede, The Netherlands \\ (Received 29 December 2006; published 7 August 2007)
}

\begin{abstract}
We predict that the magnetization direction of a ferromagnet can be reversed by the spin-transfer torque accompanying spin-polarized thermoelectric heat currents. We illustrate the concept by applying a finiteelement theory of thermoelectric transport in disordered magnetoelectronic circuits and devices to metallic spin valves. When thermalization is not complete, a spin heat accumulation vector is found in the normal-metal spacer, i.e., a directional imbalance in the temperature of majority and minority spins.

PACS numbers: 72.15.Jf, 75.30.Sg, 75.60.Jk, 85.75.-d
\end{abstract}

Spintronics seeks to exploit the interplay of conduction electron charge and spin transport in nanostructures. The modulation of an electric current in a spin valve, i.e., a normal-metal spacer sandwiched between two ferromagnets, by the relative magnetization directions is the essence of the celebrated giant magnetoresistance (GMR) effect. Magnetization reversal by the current-induced spintransfer torque in spin valves or tunnel junction [1] has already been applied in memory devices as a low-power alternative to Oersted-field magnetization switching [2].

Increasing data storage density and access rate is a continuing challenge for the magnetic recording industry. The relatively high current densities and voltages that are required to operate magnetic random access memories give rise to heating effects that complicate modeling and deteriorate device stability and lifetime, making it difficult to reduce device sizes. Controlled heating can, however, also be beneficial: recording by thermally assisted reversal of magnetization via short laser pulses [3] or by Joule heating at highly resistive thin layers [4] is a possible solution for the next generation of high-density nonvolatile data storage.

Johnson and Silsbee [5] and Wegrowe [6] analyzed the thermodynamics of transport in collinear ferromagneticnormal-metal heterostructures in the diffuse regime. The measured magnetothermoelectric power and Peltier effect of multilayered nanowires has been described in terms of spin-dependent and spin-flip processes in the bulk layers by Gravier et al. [7]. A large Peltier cooling effect in transition metal nanopillars has been measured by Fukushima et al. [8]. Nonlinear thermoelectric transport in noncollinear magnetic tunnel junctions has been studied numerically in a tight-binding approximation [9]. Tsyplyatyev et al. invoked thermally excited spin currents [10] to explain thermomagnetic effects in metals with embedded ferromagnetic clusters [11]. In this Letter we report another example of "spin caloritronics," viz. a strong coupling of thermoelectric spin and charge transport with the magnetization dynamics in nanoscale magnetic structures. We establish the existence of a thermally induced torque on the magnetization at an interface between a normal metal and a ferromagnet. The conditions that should be met in order to observe this phenomenon experimentally are discussed in detail for disordered transition metal-based ferromagnet|normal metal|ferromagnet spin valves which opens the possibility to switch magnetizations by, e.g., pulsed laser heating.

In bulk metallic systems, electron transport is well described by semiclassical diffusion theory [12]. However, atomically sharp interfaces should be treated using quantum mechanical scattering matrices [13]. In mesoscopic systems such as quantum point contacts [14] scattering theory is a well established framework for understanding thermoelectric transport [15]. Here we treat magnetic nanostructures by assuming electronic distribution functions in "bulk" layers that are connected with boundary conditions at interfaces in terms of microscopic scattering matrices using an extension of a finite-element (or circuit) theory [16-18] to treat charge, spin, and energy currents on an equal footing. Interface scattering is parametrized by a few material-specific conductances that are accessible to first-principles calculations.

We start by partitioning a conducting structure into discrete low-resistance nodes connected by resistive elements. Ferromagnetic $(F)$ or normal-metal $(N)$ nodes are characterized by $2 \times 2$ distribution matrices in spin space that can be expanded into a scalar and a vector component $\hat{f}^{F(N)}=f_{c}^{F(N)} \hat{1}+\hat{\boldsymbol{\sigma}} \cdot \mathbf{s}^{F(N)} f_{s}^{F(N)}$. The unit vector of the spin quantization axis $\mathbf{s}^{F}$ is parallel to the magnetization of the ferromagnet, whereas $\mathbf{s}^{N}$ can point in any direction. An imbalance between the distribution functions at two neighboring nodes induces a nonequilibrium current. In linear response, the $2 \times 2$ spectral current in spin space across a ferromagnet-normal-metal junction at energy $\epsilon$ in the absence of spin flip and inelastic scattering is given by Ohm's law [17] 


$$
\hat{\imath}_{N \mid F}(\boldsymbol{\epsilon})=\sum_{\alpha \beta} G^{\alpha \beta}(\boldsymbol{\epsilon}) \hat{u}^{\alpha}\left[\hat{f}^{F}(\boldsymbol{\epsilon})-\hat{f}^{N}(\boldsymbol{\epsilon})\right] \hat{u}^{\beta},
$$

where $\hat{u}^{\dagger(l)}=(\hat{1} \pm \hat{\boldsymbol{\sigma}} \cdot \mathbf{m}) / 2$ are projection matrices in which $\hat{1}$ is the $2 \times 2$ unit matrix, the unit vector $\mathbf{m}$ denotes magnetization direction in the ferromagnet, and $\hat{\boldsymbol{\sigma}}$ is the vector of Pauli matrices. The conductance tensor elements read $G^{\alpha \beta}=\left(e^{2} / h\right) \sum_{n m}\left[\delta_{m n}-r_{n m}^{\alpha}\left(r_{n m}^{\beta}\right)^{*}\right]$ in terms of the energy-dependent reflection coefficients $r_{n m}^{\alpha}(\epsilon)$ for majority and minority spins at the $N \mid F$ interface. The total charge and heat spin-matrix currents are defined as $\hat{I}=$ $\int d \epsilon \hat{\imath}(\epsilon)$ and $e \hat{\dot{Q}}=\int d \epsilon(\epsilon-\mu) \hat{\imath}(\epsilon)=e \hat{I}^{\varepsilon}-\mu \hat{I}$, respectively, where $\mu$ is the equilibrium chemical potential and $\hat{I}^{\varepsilon}$ the energy current. The charge and spin electric currents $I_{c}$ and $\mathbf{I}_{s}$ are the scalar and vector components of the matrix current $\hat{I}=\left(I_{c} \hat{1}+\hat{\boldsymbol{\sigma}} \cdot \mathbf{I}_{s}\right) / 2$. Analogously, $\hat{\dot{Q}}=$ $\left(\dot{Q}_{c} \hat{1}+\hat{\boldsymbol{\sigma}} \cdot \dot{\mathbf{Q}}_{s}\right) / 2$.

When inelastic scattering in a given node is weak, the concept of a local temperature is not applicable and the distribution function has to be determined as a function of energy [19], as will be discussed in a future publication. Here we assume either that the applied voltage is much smaller than the temperature or that there is sufficient inelastic scattering so that $f_{\uparrow(\downarrow)}=f_{c(-)}^{+} f_{s}$ may be parametrized by Fermi-Dirac distribution functions with spindependent chemical potentials $\mu_{\uparrow(\downarrow)}=\mu-e V_{\uparrow(\downarrow)}$ and temperatures $T_{\uparrow(\downarrow)}$ that are weakly perturbed from their values at equilibrium $(\mu, T)$. When conductances do not vary too rapidly in an energy interval $k_{B} T$ around the Fermi level, Sommerfeld's expansion of the distribution functions up to order $\left(k_{B} T / \mu\right)^{2}$ may be invoked, where $k_{B}$ is Boltzmann's constant [20]. Defining charge and spin temperatures $T_{c}=$ $\left(T_{\uparrow}+T_{\downarrow}\right) / 2$ and $T_{s}=T_{\uparrow}-T_{\downarrow}$ (similarly for $\mu_{c}$ and $\mu_{s}, V_{c}$ and $V_{s}$ ), we also require $T_{s} \ll 2 T_{c}$ in the following. The

$$
\left(\begin{array}{c}
\mathbf{I}_{s}^{\perp} \\
\dot{\mathbf{Q}}_{s}^{\perp}
\end{array}\right)=\left(\begin{array}{c}
\operatorname{Re} G^{\uparrow \downarrow} \mathbf{m} \times+\operatorname{Im} G^{\uparrow \downarrow} \\
e L_{0} T^{2}\left(\operatorname{Re} G_{\epsilon}^{\uparrow \downarrow} \mathbf{m} \times+\operatorname{Im} G_{\epsilon}^{\uparrow \downarrow}\right)
\end{array}\right.
$$

which are parametrized by the (spin-)mixing conductance $G^{\dagger \downarrow}$ and its energy derivative $G_{\epsilon}^{\dagger \downarrow}=\left.\partial_{\epsilon} G^{\dagger \downarrow}\right|_{\epsilon_{F}}$. We disregard in the following the imaginary part of the mixing conductance [18] and its energy derivative. In analogy with the dimensionless mixing conductance $\eta=2 \operatorname{Re} G^{\dagger \downarrow} / G$ we also introduce a dimensionless "mixing thermopower" as $\eta^{\prime}=2 \operatorname{Re} G_{\epsilon}^{\dagger \downarrow} / G_{\epsilon}$. Both transverse spin currents $\mathbf{I}_{s}^{\perp}$ and $\dot{\mathbf{Q}}_{s}^{\perp}$ are absorbed by the ferromagnet and transferred as a torque on the magnetization order parameter.

We extend the methodology used to calculate bare interface conductances at the Fermi energy [21] to obtain its energy dependence $G^{\alpha \beta}(\epsilon)$. A finite drift is taken into account by replacing $G^{\alpha \beta}(\epsilon)^{-1}$ with $G^{\alpha \beta}(\epsilon)^{-1}-$ $\left(h / 2 e^{2}\right)\left[N^{N}(\epsilon)^{-1}+\delta_{\alpha \beta} N^{\alpha F}(\epsilon)^{-1}\right] \quad[13,18], \quad$ where $N^{\alpha F}(\epsilon)$ is the number of propagating modes of spin $\alpha$ at
Sommerfeld expansion leads to integrals of the form $\int d \epsilon(\epsilon-\mu)^{d} f_{s}(\epsilon)$ that for $d=0,1,2$ read $\mu_{s}$, $\left(\pi^{2} k_{B}^{2} / 3\right) T T_{s}$ and $\left(\pi^{2} k_{B}^{2} / 3\right) T^{2} \mu_{s}$, respectively. The same integrals over the function $f_{c}^{F}-f_{c}^{N}$ result in similar expressions by $\mu_{s} \rightarrow \mu_{c}^{F}-\mu_{c}^{N}$ and $T_{s} \rightarrow T_{c}^{F}-T_{c}^{N}$. The spin and heat currents through an $N \mid F$ interface are spanned by longitudinal components polarized parallel to $\mathbf{m}\left(I_{s}^{\|}=\mathbf{m} \cdot \mathbf{I}_{s}\right.$ and $\left.\dot{Q}_{s}^{\|}=\mathbf{m} \cdot \dot{\mathbf{Q}}_{s}\right)$ and transverse contributions $\mathbf{I}_{s}^{\perp}=\mathbf{I}_{s}-I_{s}^{\|} \mathbf{m}$ and $\dot{\mathbf{Q}}_{s}^{\perp}=\dot{\mathbf{Q}}_{s}-\dot{Q}_{s}^{\|} \mathbf{m}$. The matrix that relates the particle, heat, and spin currents is equivalent to those found in the literature [5,7] when m || s:

$$
\begin{aligned}
\left(\begin{array}{c}
I_{c} \\
\dot{Q}_{c} \\
I_{s}^{\|} \\
\dot{Q}_{s}^{\|}
\end{array}\right)= & G\left(\begin{array}{cccc}
1 & -S & P & -P^{\prime} S \\
-S T & L_{0} T & -P^{\prime} S T & P L_{0} T \\
P & -P^{\prime} S & 1 & -S \\
-P^{\prime} S T & P L_{0} T & -S T & L_{0} T
\end{array}\right) \\
& \times\left(\begin{array}{c}
-\left(V_{c}^{F}-V_{c}^{N}\right) \\
T_{c}^{F}-T_{c}^{N} \\
-\left(V_{s}^{F}-\mathbf{m} \cdot \mathbf{s} V_{s}^{N}\right) / 2 \\
\left(T_{s}^{F}-\mathbf{m} \cdot \mathbf{s} T_{s}^{N}\right) / 2
\end{array}\right),
\end{aligned}
$$

where $G=G^{\dagger}+G^{\downarrow}$ is the total conductance, $S=$ $-\left.e L_{0} T \partial_{\epsilon} \ln G\right|_{\epsilon_{F}}$ is the thermopower (Mott's law), both at the Fermi energy $\left(\epsilon_{F}\right)$, and $L_{0}=\left(\pi k_{B} / e\right)^{2} / 3 \simeq 2.45 \times$ $10^{-8} \mathrm{~W} \Omega \mathrm{K}^{-2}$ is the Lorenz number. $P=\left(G^{\dagger}-G^{\downarrow}\right) / G$ is the polarization of the conductance with $|P| \leq 1$ and $P^{\prime}=$ $\left.\partial_{\epsilon}\left(G^{\uparrow}-G^{\downarrow}\right)\right|_{\epsilon_{F}} /\left.\partial_{\epsilon} G\right|_{\epsilon_{F}}$ is the polarization of its energy derivative at the Fermi energy. In contrast to $P,\left|P^{\prime}\right|$ is not bounded and $P^{\prime} S$ can be very large, e.g., when a van Hove singularity is close to the Fermi energy for one spin direction. $P_{S}=\left(S_{\uparrow}-S_{\downarrow}\right) /\left(S_{\uparrow}+S_{\downarrow}\right)=\left(P^{\prime}-P\right) /(1-$ $\left.P^{\prime} P\right)$ is the spin polarization of the thermopower. We focus here on the transverse spin currents:

$\left.\begin{array}{c}e L_{0} T\left(\operatorname{Re} G_{\epsilon}^{\dagger} \mathbf{m} \times+\operatorname{Im} G_{\epsilon}^{\dagger \downarrow}\right) \\ L_{0} T\left(\operatorname{Re} G^{\dagger} \mathbf{m} \times+\operatorname{Im} G^{\dagger \downarrow}\right)\end{array}\right)\left(\begin{array}{c}V_{s}^{N} \mathbf{s} \times \mathbf{m} \\ -T_{s}^{N} \mathbf{s} \times \mathbf{m}\end{array}\right)$,

energy $\epsilon$ in $F$. To determine the thermopower $\ln G^{\alpha \beta}(\epsilon)$ is differentiated numerically. The results for $S, P^{\prime}, P_{S}, \eta$, and $\eta^{\prime}$ are listed for a number of important interfaces in Table I. Note that the spin polarization of the thermopower in bulk magnets, believed to be dominated by electron-magnon spin-flip scattering $[7,22]$, has a different origin.

The temperature $T_{c}$, voltage $V_{c}$, particle spin accumulation $\mathbf{s} V_{s}$ and temperature spin accumulation $\mathbf{s} T_{s}$ of a given node are governed by Kirchhoff rules. Charge and angular momentum conservation implies that the sums of all charge and all spin currents flowing into a given node vanish, respectively. Since thermal transport in metals is dominated by the conduction electrons [23] we disregard the phonon contribution to the energy currents. Electrons experience inelastic electron-electron and electron-phonon 
TABLE I. Thermoelectric interface parameters calculated at the Fermi energy for a number of almost lattice-matched interfaces including a drift correction $[13,18]$. The star $*$ indicates a dirty interface modeled in a $10 \times 10$ lateral supercell with two layers of $50 \%-50 \%$ alloy.

\begin{tabular}{lcrrrr}
\hline \hline & $\frac{S}{T}\left(\mathrm{nV} / \mathrm{K}^{2}\right)$ & $P^{\prime}(\%)$ & $P_{S}(\%)$ & $\eta$ & $\eta^{\prime}$ \\
\hline $\mathrm{Cu} / \mathrm{Co}(001)$ & -13 & 72 & -8 & 0.50 & 0.03 \\
$\mathrm{Cu} / \mathrm{Co}(001)^{*}$ & -34 & 89 & 43 & 0.49 & 0.06 \\
$\mathrm{Cu} / \mathrm{Co}(110)$ & -10 & 6 & -66 & 0.67 & -0.32 \\
$\mathrm{Cu} / \mathrm{Co}(110)^{*}$ & -13 & 85 & 45 & 0.63 & 0.07 \\
$\mathrm{Cu} / \mathrm{Co}(111)$ & -15 & 56 & -6 & 0.53 & 0.13 \\
$\mathrm{Cu} / \mathrm{Co}(111)^{*}$ & -15 & 77 & 17 & 0.64 & 0.13 \\
$\mathrm{Cr} / \mathrm{Au}(001)$ & 7 & 0 & 0 & $\ldots$ & $\ldots$ \\
$\mathrm{Cr} / \mathrm{Au}(001)^{*}$ & 0 & 0 & 0 & $\ldots$ & $\ldots$ \\
$\mathrm{Cr} / \mathrm{Fe}(001)$ & 22 & -40 & 48 & 4.23 & -4.27 \\
$\mathrm{Cr} / \mathrm{Fe}(001)^{*}$ & 7 & -190 & -9500 & 3.25 & -0.48 \\
$\mathrm{Cr} / \mathrm{Co}(001)$ & 62 & -111 & -160 & 3.03 & -2.86 \\
$\mathrm{Cr} / \mathrm{Co}(001)^{*}$ & 23 & -95 & -92 & 2.92 & -0.86 \\
\hline \hline
\end{tabular}

collisions. $T_{s}$ decays then by the energy exchange between the electrons. The spin accumulation $V_{s}$ is dissipated to the lattice by spin-flip scattering which can be very weak in selected metals and is disregarded here for simplicity. We distinguish two different regimes by comparing the dwell time $\tau_{d}=e^{2} \mathcal{D} /(4 \pi G)$, where $\mathcal{D}$ is the density of states, with $\tau_{E}$, the energy relaxation time: electrons are completely thermalized when $\tau_{E} \ll \tau_{d}$, but effectively noninteracting in the opposite regime $\tau_{E} \gg \tau_{d}$. The electron dwell time in metallic nanopillars with a spacer thickness of $10 \mathrm{~nm}$ can be estimated to be $\sim 100 \mathrm{fs}$. At low temperatures this can be much shorter than either electron-electron or electron-phonon scattering times [24] and the spin temperature difference or spin heat accumulation becomes an important parameter. For elevated temperatures inelastic scattering is more effective and we adopt a complete thermalization model.

We illustrate the theory for symmetric $F_{L}\left(\mathbf{m}_{1}\right)|N| F_{R}\left(\mathbf{m}_{2}\right)$ spin valves (see Fig. 1) consisting of two ferromagnetic reservoirs separated by a normal-metal node via two resistive contacts with variable magnetization directions. We calculate the electric particle and heat currents and the spin-transfer torques for a voltage bias $\Delta V=$ $V_{R}-V_{L}$ and temperature bias $\Delta T=T_{R}-T_{L}$ in the thermalized as well as noninteracting regimes.

In the thermalized regime the spin heat accumulation vanishes, $T_{s}^{N(F)}=0$. In the steady state, lattice and electron temperature are the same and energy is conserved in the node's electronic system. We find for the total electric current as a function of the angle $\theta$ between the two magnetizations

$I_{c}=\frac{G}{2}(\Delta V+S \Delta T)-\frac{P G}{2} \frac{\tan ^{2} \theta / 2}{\eta+\tan ^{2} \theta / 2}\left(P \Delta V+P^{\prime} S \Delta T\right)$.

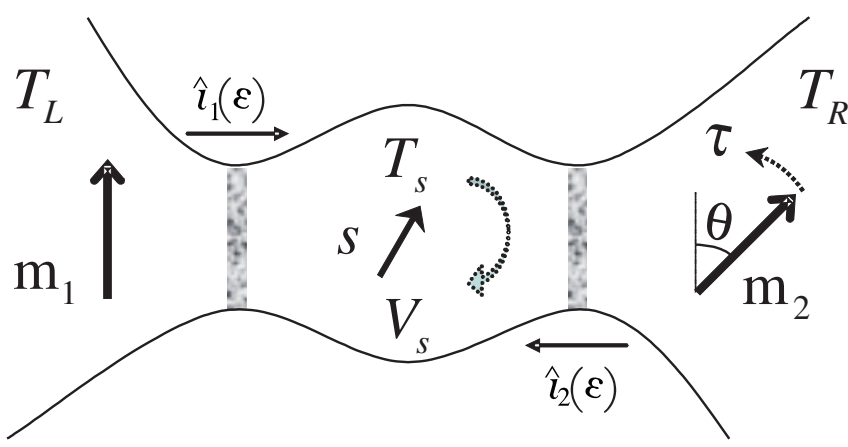

FIG. 1 (color online). Schematic of a noncollinear $F\left(\mathbf{m}_{1}\right)|N| F\left(\mathbf{m}_{2}\right)$ spin valve. Spin-dependent resistive elements separate the ferromagnetic reservoirs and the normal-metal node. A temperature bias induces a spin accumulation in the form of heat and angular momentum imbalance, the interplay of which is governed by inelastic scattering.

The angular magnetoresistance for $\Delta T=0$, measured by Urazhdin et al. [25], is well described by circuit theory [26]. When the current bias vanishes, a temperature bias $\Delta T$ induces an angular magnetothermopower $\Delta V$ that depends on both $P$ and $P^{\prime}$

$$
\left(\frac{-\Delta V}{S \Delta T}\right)_{I=0}=\frac{\eta+\left(1-P P^{\prime}\right) \tan ^{2} \theta / 2}{\eta+\left(1-P^{2}\right) \tan ^{2} \theta / 2}
$$

The angular dependence of the heat current

$$
\begin{aligned}
\dot{Q}_{c}= & -\Pi \frac{G}{2}(\Delta V+S \Delta T)-\frac{\kappa}{2} \Delta T \\
& +\Pi \frac{P^{\prime} G}{2} \frac{\tan ^{2} \theta / 2}{\eta+\tan ^{2} \theta / 2}\left(P \Delta V+P^{\prime} S \Delta T\right)
\end{aligned}
$$

where $\Pi=S T$ is the interface Peltier coefficient, strongly violates the Wiedemann-Franz law $\left(\kappa \approx L_{0} T G\right)$. A nonnegative entropy production rate in the Sommerfeld approximation requires $\left|S_{\max }\right|=\sqrt{L_{0}} \simeq 157 \mu \mathrm{V} / \mathrm{K}$ [27].

The spin-transfer torque exerted on the magnetizations by a temperature difference over the spin valves in the thermalized electron regime reads $\left(\tau=\tau_{\Delta V}+\tau_{\Delta T}\right)$

$$
\tau=\frac{G}{2} \frac{\eta \sin \theta}{\eta(1+\cos \theta)+(1-\cos \theta)}\left(P \Delta V+P^{\prime} S \Delta T\right)
$$

We can understand the similarity of the torque induced by the voltage and temperature bias as follows. A temperature difference over the spin valves initially induces different temperatures for the spin species in the normal metal node. Since we consider here the strongly interacting regime, such a temperature difference relaxes quickly due to collisions that exchange energy between spin systems but conserve the total energy. This is possible only by generating a spin current and accumulation that subsequently induces a torque just as the voltage does.

The dynamics of the magnetic layers is governed by a Landau-Lifshitz-Gilbert equation augmented by the spin- 
transfer torque. We use Slonczewski's estimate for the critical current [1] that leads to magnetization reversal in metallic ferromagnets, replacing $P \Delta V$ with $P^{\prime} S \Delta T$. A thermoelectric voltage $S \Delta T \sim 100 \mu \mathrm{V}$ corresponds to typical switching current densities of $10^{7} \mathrm{~A} \mathrm{~cm}^{-2}$. Assuming that a laser pulse provides local heating corresponding to $\Delta T \sim 100 \mathrm{~K}$, we require $P^{\prime} S \sim 1 \mu \mathrm{V} / \mathrm{K}$, which is not an unrealistic value at room temperature (see Table I). When the magnetic layers become thicker, the bulk resistance and thermopower of the layers dominate. The series resistor rule $S / G \simeq \sum_{i} S_{i} / G_{i}$, where $G_{i}$ and $S_{i}$ account for both bulk layers and interfaces in a multilayer structure, holds for $P_{S_{i}} \ll 1$. Using the bulk parameters by Gravier et al. [28] we estimate that the effective thermopower can be much higher than a $\mu \mathrm{V} / \mathrm{K}$, implying a strongly increased relative efficiency of thermal magnetization reversal $\tau_{\Delta T} / \tau_{\Delta V}$ for thicker magnetic layers. The conditions for thermal spin-torque switching are presumably more easily met in spin valves based on magnetic semiconductors [29].

In the absence of energy relaxation in the normal node, a vector spin heat accumulation $\mathbf{s} T_{s}^{N}$ develops. When $S^{2} \ll$ $L_{0}$, the "noninteracting" thermal spin-transfer torque $\tau_{\Delta T}^{*}$ reduces to the simple expression

$$
\left.\frac{\tau_{\Delta T}^{*}-\tau_{\Delta T}}{\tau_{\Delta T}}\right|_{\Delta V=0}=\frac{\left(\eta^{\prime}-\eta\right) P}{\eta P^{\prime}} \frac{\tan ^{2} \theta / 2}{\eta+\tan ^{2} \theta / 2} .
$$

The sensitivity of the thermal spin-transfer torque to interaction effects vanishes for spin valves with half-metallic ferromagnets in which $\eta=\eta^{\prime}=2$. The spin heat accumulation becomes

$$
\left.\frac{T_{s}^{N}}{\Delta T}\right|_{\Delta V=0}=\frac{\eta P^{\prime}}{\left(\eta^{\prime}-\eta\right) \sin \theta / 2} \frac{\tau_{\Delta T}^{*}-\tau_{\Delta T}}{\tau_{\Delta T}} .
$$

In conclusion, we presented a circuit theory of thermoelectric transport in noncollinear spin valves. In thinly layered structures, transport properties are governed by interface conductances and their energy derivatives that have been computed from first principles. We predict a spin-transfer torque associated with purely thermal currents that can be large enough to reverse magnetizations. The concepts of spin heat accumulation and spin-mixing thermopower have been introduced to describe the thermoelectric transport in different energy relaxation regimes. We expect that a temperature gradient can excite magnetization dynamics in magnetic tunnel junctions and domain walls in ferromagnetic wires as well.

We acknowledge helpful discussions with A. Brataas, Y. Tserkovnyak, A. Fukushima, Y. Suzuki, S. Yuasa, A. Deac, X. Waintal, and H. Pothier. This work has been supported by NanoNed, EC Contracts No. IST-033749
"DynaMax" and No. NMP-505587-1 "SFINX," the "Stichting voor Fundamenteel Onderzoek der Materie" (FOM) and the "Stichting Nationale Computer Faciliteiten" (NCF).

[1] J. C. Slonczewski, J. Magn. Magn. Mater. 159, L1 (1996); L. Berger, Phys. Rev. B 54, 9353 (1996); M. D. Stiles and J. Miltat, Top. Appl. Phys. 101, 225 (2006).

[2] M. Hosomi et al., IEDM Technical Digest (IEEE, Piscataway, NJ, 2005), p. 473.

[3] T. W. McDaniel, J. Phys. Condens. Matter 17, R315 (2005).

[4] I. L. Prejbeanu et al., IEEE Trans. Magn. 40, 2625 (2004).

[5] M. Johnson and R.H. Silsbee, Phys. Rev. B 35, 4959 (1987); M. Johnson, J. Supercond. 16, 679 (2003).

[6] J.-E. Wegrowe, Phys. Rev. B 62, 1067 (2000).

[7] L. Gravier et al., Phys. Rev. B 73, 024419 (2006); 73, 052410 (2006).

[8] A. Fukushima et al., IEEE Trans. Magn. 41, 2571 (2005).

[9] Z. C. Wang, G. Su, and S. Gao, Phys. Rev. B 63, 224419 (2001).

[10] O. Tsyplyatyev, O. Kashuba, and V. I. Fal'ko, Phys. Rev. B 74, 132403 (2006).

[11] S. Serrano-Guisan et al., Nat. Mater. 5, 730 (2006).

[12] F. J. Jedema, A. T. Filip, and B. J. van Wees, Nature (London) 410, 345 (2001); F. J. Jedema et al., Nature (London) 416, 713 (2002).

[13] K. M. Schep et al., Phys. Rev. B 56, 10805 (1997).

[14] L. W. Molenkamp et al., Phys. Rev. Lett. 68, 3765 (1992).

[15] P. N. Butcher, J. Phys. Condens. Matter 2, 4869 (1990).

[16] Yu. V. Nazarov, Phys. Rev. Lett. 73, 134 (1994).

[17] A. Brataas, Yu. V. Nazarov, and G. E. W. Bauer, Phys. Rev. Lett. 84, 2481 (2000); Eur. Phys. J. B 22, 99 (2001).

[18] A. Brataas, G. E. W. Bauer, and P. J. Kelly, Phys. Rep. 427, 157 (2006).

[19] F. Pierre, Ann. Phys. (Paris) 26, 1 (2001).

[20] N. W. Ashcroft and N.D. Mermin, Solid State Physics (Saunders, Philadelphia, 1976).

[21] K. Xia et al., Phys. Rev. B 63, 064407 (2001); K. Xia et al., Phys. Rev. B 73, 064420 (2006).

[22] L. Piraux et al., J. Magn. Magn. Mater. 110, L274 (1992).

[23] B. C. Gundrum, D. G. Cahill, and R. S. Averback, Phys. Rev. B 72, 245426 (2005).

[24] H. Pothier et al., Phys. Rev. Lett. 79, 3490 (1997); B. Huard et al., Phys. Rev. Lett. 95, 036802 (2005).

[25] S. Urazhdin, R. Loloee, and W. P. Pratt, Jr., Phys. Rev. B 71, 100401(R) (2005).

[26] A. A. Kovalev, G. E. W. Bauer, and A. Brataas, Phys. Rev. B 73, 054407 (2006).

[27] G. D. Guttman, E. Ben-Jacob, and D. J. Bergman, Phys. Rev. B 52, 5256 (1995).

[28] L. Gravier et al., J. Phys. D 39, 5267 (2006).

[29] D. Chiba et al., Phys. Rev. Lett. 93, 216602 (2004). 